\title{
Perception of coherent motion in random dot displays by pigeons and humans
}

\author{
WALTER F. BISCHOF, SHERI L. REID, DOUG R. W. WYLIE, and MARCIA L. SPETCH \\ University of Alberta, Edmonton, Alberta, Canada
}

\begin{abstract}
Pigeons and humans were required to discriminate coherent from random motion in dynamic random dot displays. Coherence and velocity thresholds were determined for both species, and both thresholds were found to be substantially higher for pigeons than for humans. The results are discussed with reference to differences in motion processing in mammals and birds. It is suggested that the inferior motion sensitivity of pigeons can be attributed to poorer spatiotemporal motion integration.
\end{abstract}

Numerous adaptive behaviors are dependent on the detection of motion (Frost, Wylie, \& Wang, 1994; Nakayama, 1985). The identification of camouflaged predators, locomotion through the environment, and the recognition of species-typical movement patterns all rely on some form of motion processing. Electrophysiological studies show that most neurons in the pigeon visual system respond better to moving than to stationary stimuli (e.g., Britto, Brunelli, Francesconi, \& Magni, 1975; Frost \& DiFranco, 1976; Hughes \& Pearlman, 1974; Miceli, Gioanni, Reperant, \& Peyrichoux, 1979). Nevertheless, pigeons have typically been described as poor motion detectors (e.g., Hodos, Smith, \& Bonbright, 1975). However, the number of studies investigating motion processing in the pigeon is small, and differences in methodology make it difficult to compare motion detection thresholds across experiments or species. The present study provides a descriptive account of the pigeon's ability to detect motion in dynamic random dot displays and provides the first direct comparison of pigeons' performance with that of humans.

Behavioral studies suggest that movement is a dominant stimulus dimension for pigeons (Pisacreta \& Witt, 1985). Pigeons can detect apparent motion (Siegel, 1970, 1971), and can discriminate complex movement patterns consisting of Lissajous figures (Emmerton, 1986). Nevertheless, studies have shown that pigeons' absolute velocity thresholds are considerably higher than those estimated for humans (which are typically on the order of 0.17-0.33 deg/sec for peripheral vision; Johannson, 1978). For example, Hodos et al. (1975) reported that pigeons' mean velocity thresholds for movement detection were 4 and $6 \mathrm{deg} / \mathrm{sec}$ for rotating radial stripes and horizontally moving bars, respectively. Martinoya and Delius

This research was supported by research grants from the Natural Sciences and Engineering Research Council of Canada to W.F.B., D.R.W.W., and M.L.S and by an Alberta Heritage Establishment Grant to D.R.W.W. We thank D. Kelly and K. Steinbring for assistance in conducting the research and $\mathrm{B}$. Hodge for programming the motion stimuli. Correspondence concerning this article should be addressed to W. F. Bischof, Department of Psychology, University of Alberta, Edmonton, AB T6G 2E9, Canada (e-mail: wfb@ualberta.ca).
(1990), using the rotating spiral illusion, reported velocity thresholds of between 1 and $3 \mathrm{deg} / \mathrm{sec}$, which are below the velocity thresholds obtained by Hodos et al. (1975) but still above those typically reported for humans. Motion sensitivity in pigeons also appears to depend on the visual field in which the stimuli are presented. Using a moving square-wave grating as a stimulus, Martinoya, Rivaud, and Bloch (1983) found that pigeons' motion thresholds were $1.3-2.5 \mathrm{deg} / \mathrm{sec}$ in the lateral visual field, as compared with $3.6-8.8 \mathrm{deg} / \mathrm{sec}$ in the frontal visual field.

Undoubtedly, estimates of the pigeons' motion sensitivity vary substantially with the methodology employed. Hence, comparisons of motion sensitivity of pigeons and humans are somewhat premature at this point, given that the thresholds were not obtained with the same methodology. The present study contributes to our understanding of motion processing in the pigeon in three primary ways. First, similar stimuli were used to determine thresholds in both pigeons and humans, to provide as direct a comparison of motion sensitivity in the two species as possible. Second, to our knowledge, this is the first study to determine motion detection thresholds in the pigeon with dynamic random dot stimuli. The primary advantage to using these stimuli is that they eliminate the use of positional and form cues (Nakayama \& Tyler, 1981) and, thus, tap the basic motion detection mechanism. Third, we included a control experiment (Experiment 4), in which the performance of pigeons and humans was compared on a task that required a visual discrimination based on spatial frequency rather than on motion. Because the spatial frequency discrimination task was similar to the motion detection task in all respects except the stimulus dimension being discriminated, this allowed us to assess whether differences between humans and pigeons specifically reflected differences in motion detection.

\section{GENERAL METHOD}

\section{Subjects}

Eight adult silver king pigeons with various experimental histories made up the animal subjects. All the birds had prior experience in tasks using the touch screen. The birds were maintained at ap- 
proximately $85 \%$ of their free-feeding weights by mixed grain obtained during experimental sessions and supplemental feedings of pigeon chow. They were housed in large individual cages under a 12:12-h light:dark cycle (lights on at 6:00 a.m.). Water and grit were freely available in the home cages. A total of 4 human observers participated in the study, 2 of the authors and 2 naive subjects. All had normal vision. For pigeons, the order of participation in the experiments varied across subjects, as will be described in the individual experiments. For humans, the order of participation varied randomly across conditions.

\section{Apparatus}

The birds were tested in a modified rectangular pigeon chamber (BRS/LVE) with a large opening for the monitor cut into one end wall, $8 \mathrm{~cm}$ above the chamber floor. An NEC color monitor with an attached infrared touch frame (Carroll Touch 1490 smart frame) was placed against the opening. A thin sheet of Plexiglas covered the monitor screen, and the touch screen was spaced approximately $1.6 \mathrm{~cm}$ from the screen. A Plexiglas food cup was centered on the wall below the screen, and a lamp beneath the cup illuminated food presentations. A Colbourn pellet dispenser, attached to the top of the chamber, dispensed 45-mg pellets through an attached tube into the food cup. Each food presentation consisted of the delivery of two pellets and a 4-sec illumination of the food cup.

Although the pigeons were free to view the display from anywhere in the chamber, previous reports in the literature (Hodos, Leibowitz, \& Bonbright, 1976) and our own systematic observations of 3 birds responding on the motion detection task indicated that the birds almost invariably stood very close to the Plexiglas surface in front of the screen; viewing distance from the stimulus display was, therefore, approximately $9 \mathrm{~cm}$.

Microcomputers, located in an adjacent room, controlled experimental contingencies and recorded peck coordinates. The touch frames were programmed to detect individual pecks (i.e., detection of a beam break, then a return to unbroken beams before another peck would be recorded).

In the experiments with human subjects, stimuli were presented on a Hitachi Superscan Elite 21 monitor under the control of a VGA display card. Screen size was $109.7 \times 93.7 \mathrm{deg}$ at the viewing distance of $14 \mathrm{~cm}$, which was controlled by a chinrest.

\section{Stimuli}

The motion displays for humans and pigeons were matched in visual angle. They consisted of two $47.9 \times 47.9 \mathrm{deg}$ random dot displays presented side by side, separated by a gap of $29.9 \mathrm{deg}$. The stimulus display on one side consisted of random motion noise $(0 \%$ coherence): For each frame change, each dot moved by 1 pixel $(0.5 \mathrm{deg})$ in a random direction (up, down, left, or right). The stimulus display on the other side provided partially coherent motion: Some percentage of the dots moved consistently in the same direction, whereas the rest of the dots moved in a random direction. This provided the impression of a coherent motion signal embedded in a background of random motion noise. The percentage of dots moving in the same direction - that is, the coherence level of the motion display-varied in the range of $1 \%-100 \%$.

Each random dot display consisted of a sequence of frames with a total duration of $1 \mathrm{sec}$ for humans. For pigeons, the display remained on until a peck to the screen was made (typically between 1 and $3 \mathrm{sec}$ ). The displays were updated every $16.6 \mathrm{msec}$ in Experiments 1 and 2 and at a variable rate in Experiment 3. Each frame contained 200 black dots (luminance approximately $5 \mathrm{~cd} / \mathrm{m}^{2}$ ), randomly distributed on a white background (luminance $98 \mathrm{~cd} / \mathrm{m}^{2}$ ). Each dot subtended a visual angle of about $0.5 \mathrm{deg}$, and dot density was approximately $0.09 \mathrm{dots} / \mathrm{deg}^{2}$. Dot lifetime was set to either 5 frames or 200 frames, depending on the experimental conditionthat is, each dot was relocated to a random position after 5 and 200 frames, respectively (Scase, Braddick, \& Raymond, 1996).
The static displays used in Experiment 4 also consisted of two $47.9 \times 47.9 \mathrm{deg}$ displays presented side by side, separated by a gap of $29.9 \mathrm{deg}$. The stimulus on one side consisted of random dot noise, with each dot $(0.5 \times 0.5 \mathrm{deg} / \mathrm{pixel})$ being either white $\left(98 \mathrm{~cd} / \mathrm{m}^{2}\right)$ or black $\left(5 \mathrm{~cd} / \mathrm{m}^{2}\right)$ with equal probability (50\% noise). The other side consisted of a square grating with a spatial frequency of five cycles per image ( 0.1 cycles/deg) presented randomly either horizontally or vertically. The gratings were embedded in noise, using the following procedure. For a given noise probability $p$, every pixel was changed from black to white or vice versa with probability $p$. Thus for $p=0$, the grating was noise free, and for $p=$ .5 , pure noise was obtained (see, e.g., Pelli, 1990).

\section{Procedure}

The stimuli were presented in a two-alternative forced-choice paradigm. On each trial, two stimulus displays were presented side by side. For motion detection (Experiments 1-3), the negative display contained random motion noise (coherence level $=0 \%$ ), and the positive display contained motion with a fixed coherence level. Coherent movement could be in one of four directions (up, down, left, or right). The direction of motion and the side containing the positive stimulus varied randomly across trials in each session. For grating detection (Experiment 4), the negative display contained no grating ( $50 \%$ noise), and the positive display contained a grating embedded in noise. The orientation of the grating (horizontal or vertical) varied randomly across trials.

The procedure used for pigeons consisted of three phases: preliminary training, simultaneous discrimination training, and testing.

Preliminary training. When required, preliminary training consisted of one or two sessions, with a modified autoshaping procedure to establish reliable pecking to the positive display. During these sessions, each trial began with the presentation of the positive stimulus at its maximum detection value $(100 \%$ coherence and maximum velocity for the motion displays in Experiments 1-3, $0 \%$ noise for the static display in Experiment 4), randomly presented on the right or the left side of the screen. The display stayed on for $8 \mathrm{sec}$ or until a peck in the area containing the positive stimulus was recorded, and then food was presented. Trials were separated by a 50 -sec intertrial interval, during which the monitor screen was blank.

Simultaneous discrimination training. The trials began with the presentation of a start stimulus, which consisted of an $8-\mathrm{cm}$ yellow square centered on the screen. The first peck to this start stimulus terminated it and produced the stimulus display, consisting of two side by side stimuli, as described above. The stimulus display was terminated by the second peck recorded within one of the two display areas. If the trial ended with a peck to the positive stimulus, food was presented, followed by a 5 -sec intertrial interval. If the trial ended with a peck to the negative stimulus, food was not presented, and the 5 -sec intertrial interval was followed by a correction trial, in which the same stimulus conditions were repeated. The results of correction trials were never used in the determination of accuracy. Each session provided 120 trials.

For all training and baseline trials, the positive stimulus in Experiments 1-3 (motion detection) was $100 \%$ coherence and a velocity of $47.9 \mathrm{deg} / \mathrm{sec}$. In Experiment 4 (spatial frequency detection), the positive stimulus during training and baseline was $0 \%$ noise. Training continued for each bird until accuracy (percentage of correct trials) reached $85 \%$ or greater for two consecutive sessions.

Testing. The test sessions were identical to the discrimination training sessions, except that the positive stimulus was systematically manipulated to determine detection thresholds, as will be described in the individual experiments. During Experiments $2 \mathrm{~A}$ and 3 , all the birds except one ( $\mathrm{C} 201)$ received baseline sessions between test sessions. For all the birds in Experiments 1, 2B, and 4, and for bird $\mathrm{C} 201$ in Experiment $2 \mathrm{~A}$, the intervening baseline sessions were omitted, but 10 baseline trials were provided at the start of each test. 
Human observers were required to indicate which of the two random dot displays contained the positive stimulus (coherently moving dots in Experiments 1-3 and gratings in Experiment 4), using the arrow keys on the keyboard, guessing if necessary. Following the response, the next trial was initiated after an intertrial interval of $1 \mathrm{sec}$. For each level of the manipulated variables (motion coherence in Experiments 1 and 2, motion velocity in Experiment 3, and percentage of noise in Experiment 4), four blocks of 25 trials were performed, resulting in 100 trials per level.

\section{EXPERIMENT 1}

In the first experiment, coherence thresholds were determined for humans and pigeons, using dynamic random dot displays in which dot lifetime was set to five frames.

\section{Method}

Subjects. Two pigeons $(6, \mathrm{C} 229)$ had previously participated in Experiments 2 and 3, whereas one of the pigeons (C201) had no prior experience with the motion stimuli used. The human subjects consisted of 2 of the authors and 2 naive observers.

Procedure. For the pigeons, coherence thresholds were determined by systematically decreasing and increasing coherence levels of the positive stimulus until accuracy either fell below $55 \%$ (descending series) or rose above $70 \%$ (ascending series), respectively. Each test session began with 10 warm-up trials in which the positive stimulus was the motion signal containing $100 \%$ coherence. During the first series (first descending), the motion coherence of the positive stimulus was decreased in $10 \%$ steps (starting from $60 \%$ coherence) across each test session until accuracy fell below $55 \%$ correct. During the second series (first ascending), the coherence was increased in 5\% steps until accuracy rose above $70 \%$ correct. During the third series (second descending), the coherence was decreased in 5\% steps until accuracy fell below 55\% correct. During the last series (second ascending), the coherence was increased in $5 \%$ steps until accuracy rose above $70 \%$ correct.

For human observers, motion detection performance was measured for coherence levels of $1 \%, 2 \%, 3 \%, 4 \%, 5 \%, 6 \%, 8 \%, 10 \%$, $15 \%, 20 \%, 25 \%$, and $30 \%$.

Threshold estimation. For all the subjects, two thresholds were estimated from the accuracy functions obtained. Linear interpolation was used to determine the point at which these functions crossed the $70 \%$ and $55 \%$ accuracy lines. The $70 \%$ threshold was selected to represent the point at which the positive stimulus could clearly be detected but the discrimination was sufficiently difficult to result in a substantial number of errors. The $55 \%$ threshold was selected as the minimum detection threshold for the positive stimulus. For the pigeons, the functions obtained during the two ascending series were used to calculate the $70 \%$ thresholds, and the functions obtained during the two descending series were used to calculate the $55 \%$ thresholds. In each case, the values were averaged across the two determinations for each bird. For the human observers, the thresholds were calculated from the single function obtained for each subject.

\section{Results and Discussion}

For all the statistical tests, the alpha level was set to .05 . The left panel of Figure 1 shows accuracy in identifying coherent motion as a function of coherence level for the 4 human observers. The $70 \%$ coherence thresholds were $5.2 \%, 1.9 \%, 6.1 \%$, and $7.2 \%$, and the $55 \%$ thresholds were $1.3 \%, 1.1 \%, 3.3 \%$, and $2.0 \%$, for the 4 observers, respectively.
The right panel of Figure 1 shows accuracy in identifying coherent motion as a function of coherence level for the 3 pigeon observers. The $70 \%$ thresholds were $31 \%$, $34 \%$, and $60 \%$, and the $55 \%$ thresholds were $22 \%, 13.5 \%$, and $21 \%$, for the 3 pigeons, respectively.

Coherence thresholds for the pigeons were, on average, eight times higher than those for the human observers. The results, thus, support previous claims that pigeons' motion sensitivity is much poorer than that of the human visual system. In our case, we can, however, provide stronger support for this claim, given that the motion sensitivity of pigeons and of humans was assessed with stimuli that were made to be as similar as possible across species.

The pigeons' accuracy did not vary systematically with direction of motion (up, down, left, and right). Accuracy for each direction of motion was compared, using the two sessions that completed the ascending thresholds (overall accuracy on these sessions was between $70 \%$ and $85 \%$ correct and, therefore, did not suffer from floor or ceiling effects). An analysis of variance (ANOVA) showed no significant differences in accuracy across the four directions of motion $[F(3,6)=1.68]$.

Given that the pigeons had unlimited time to view the display before choosing, we examined their latencies (time from onset of the motion display until the bird completed the two-peck response requirement), to determine whether viewing time varied systematically as a function of the difficulty of the discrimination. The top panel of Table 1 shows mean latencies and accuracy scores, averaged across the two sessions that completed the ascending thresholds and the two sessions that completed the descending thresholds. Despite the difference in accuracy, latencies were not significantly different for the two types of sessions $[t(2)=$ $0.60]$.

It has been suggested that the pigeons' motion sensitivity in this experiment may be lower because pigeons have a higher flicker sensitivity (critical flicker fusion up to $145 \mathrm{~Hz}$; Powell, 1967) than humans and sensitivity to motion stimuli embedded in flicker is known to be reduced (Bedell \& Johnson, 1995). This is unlikely to be a factor in the present experiment, because the flicker sensitivities of pigeons and humans are approximately equal for the background intensity $\left(98 \mathrm{~cd} / \mathrm{m}^{2}\right)$ used in this experiment (Emmerton, 1983b; Watson, 1986).

\section{EXPERIMENT 2A}

Clearly, pigeons are inferior to humans in their ability to detect coherently moving dots embedded in dynamic noise. This is not likely to be due solely to an inferiority in simple motion detection, given that performance was virtually perfect at a coherence level of $100 \%$. Rather, the source of lower motion sensitivity is probably at the level of motion integration.

For the stimuli used in Experiment 1, dot lifetime was set to five frames - that is, every coherently moving dot 


\section{Humans}
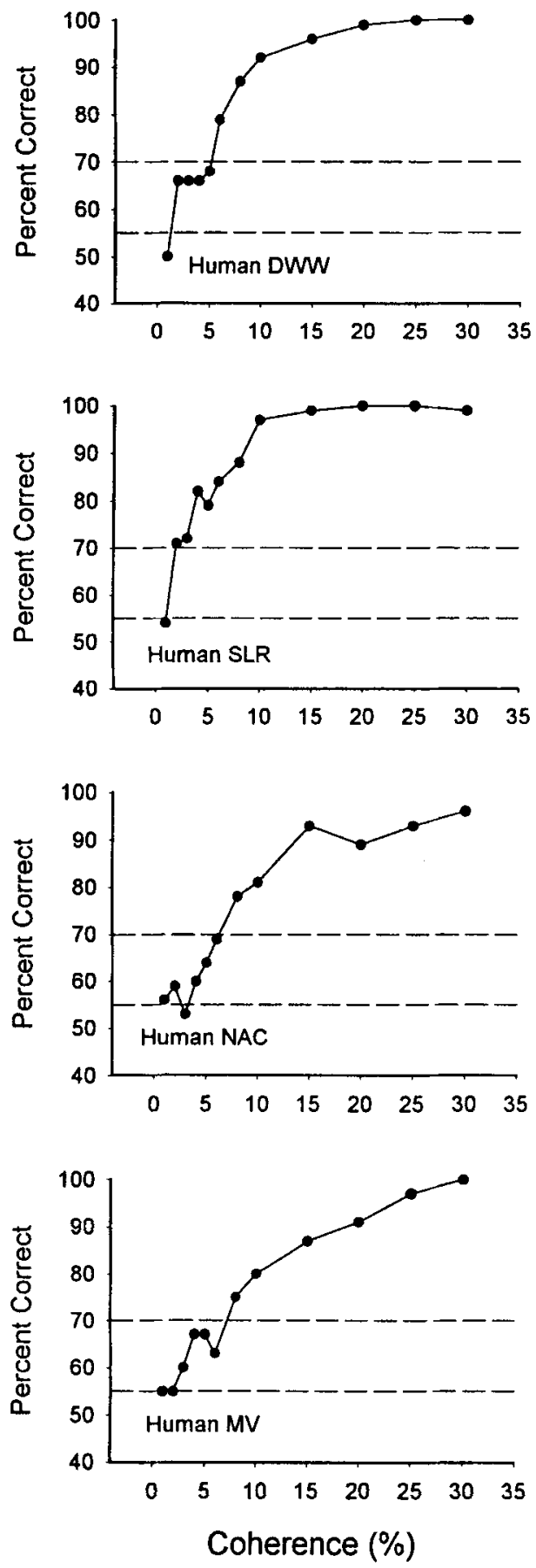

\section{Pigeons}
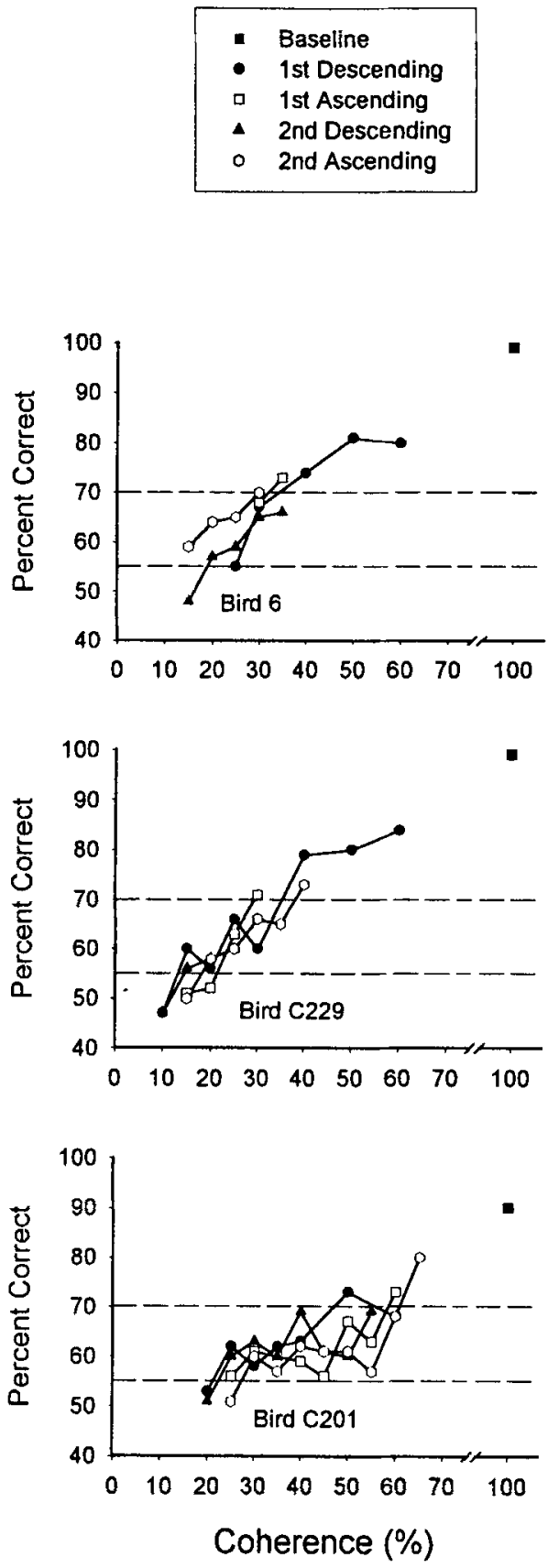

Figure 1. Coherence thresholds (see definition in text) for random dot patterns with a dot lifetime of five, for 4 human observers and 3 pigeon observers in Experiment 1.

disappeared after five frames $(83.3 \mathrm{msec})$ and reappeared at another, randomly chosen location. Thus, coherent motion signals were generated over brief temporal intervals (five frames) and, at low coherence levels, at widely spaced locations. At a local level, a single coherently moving dot stimulates (over the period of five frames) the same or, at least, closely coupled motion detectors. Local spatial and temporal integration of this stimulation is known to increase human motion sensitivity substantially (cf. hetero recruitment of motion signals; Snowden \& Braddick, 1989). The spatially distributed motion signals generated for each coherently moving dot 
Table 1

Mean (and Standard Error of the Mean) Latencies (in Milliseconds) and Accuracy Scores (\% Correct) for Pigeons in Experiments 1, 2A, 3, and 4, Averaged Across the Two Sessions That Met the Ascending Threshold and the Two Sessions That Met the Descending Threshold

\begin{tabular}{|c|c|c|c|c|c|c|c|c|}
\hline \multirow[b]{3}{*}{ Experiment } & \multicolumn{4}{|c|}{ Ascending } & \multicolumn{4}{|c|}{ Descending } \\
\hline & \multicolumn{2}{|c|}{ Latency } & \multicolumn{2}{|c|}{$\%$ Correct } & \multicolumn{2}{|c|}{ Latency } & \multicolumn{2}{|c|}{$\%$ Correct } \\
\hline & $M$ & $S E$ & $M$ & $S E$ & $M$ & $S E$ & $M$ & $S E$ \\
\hline 1 & 1,725 & 112 & 74 & 1.7 & 1,769 & 106 & 50 & 1.7 \\
\hline $2 \mathrm{~A}$ & 2,001 & 263 & 73 & 0.7 & 1,641 & 110 & 53 & 0.5 \\
\hline 3 & 1,622 & 172 & 80 & 0.8 & 1,628 & 134 & 50 & 1.5 \\
\hline 4 & 1,203 & 216 & 75.5 & 0.9 & 1,293 & 220 & 50.9 & 1.0 \\
\hline
\end{tabular}

must be spatially integrated at a larger (global) scale. Global integration of these motion signals also increases motion sensitivity (cf. homo recruitment of motion signals; Snowden \& Braddick, 1989).

The inferior motion sensitivity of the pigeon visual system could be caused by a deficiency in local motion integration, in global motion integration, or in both. The stimuli of Experiment 1 do not provide sufficient information to decide between these possibilities. However, with minimal variation in the stimuli, we are able to investigate pigeons' ability to do local motion integration and to compare it with that of humans. With a dot lifetime of 200 frames, each coherently moving dot remains in view for a much longer duration (up to $3.3 \mathrm{sec}$, limited, of course, by the total display duration). If local motion integration mechanisms are efficient, performance should increase substantially over that in Experiment 1.

\section{Method}

Subjects. The pigeon observers included the 3 pigeons from Experiment 1 and 1 additional pigeon $(\mathrm{C} 221)$ with no prior experience in motion detection tasks. Birds 6 and $\mathrm{C} 229$ also had no prior experience at motion detection (they participated in this experiment prior to serving in Experiment 1), whereas Bird C201 participated in this experiment after completing Experiment 1 . The human subjects consisted of 2 of the authors and 2 naive observers.

Procedure. Coherence thresholds with dot lifetimes of 200 frames were determined for humans and pigeons in the same manner as that in Experiment 1.

\section{Results and Discussion}

For all the statistical tests, the alpha level was set to .05 . The left panel of Figure 2 shows performance in identifying coherent motion as a function of coherence level for the 4 human observers. The $70 \%$ coherence threshold was less than $1.2 \%$ for all the observers - that is, in $70 \%-80 \%$ of the trials, a pair of coherently moving dots was detected among the 200 moving dots.

The right panel of Figure 2 shows performance in identifying coherent motion as a function of coherence level for the 4 pigeons. The $70 \%$ threshold was $22 \%, 21 \%$, $32.5 \%$, and $29.5 \%$, and the $55 \%$ threshold was $8.5 \%$, $8.5 \%, 5.5 \%$, and $11 \%$, for the 4 pigeons, respectively.

The pigeons' accuracy did not vary systematically with direction of motion (up, down, left, and right). Ac- curacy for each direction of motion was compared, using the two sessions that completed the ascending thresholds. An ANOVA showed no significant differences in accuracy across the four directions of motion $[F(3,9)=1.61]$.

In addition, the pigeons' latencies (time from onset of the motion display until the bird completed the two-peck response requirement) did not vary systematically with accuracy. The second panel of Table 1 shows mean latencies and accuracy scores, averaged across the two sessions that completed the ascending thresholds and the two sessions that completed the descending thresholds. Despite the difference in accuracy, latencies were not significantly different for the two types of sessions $[t(3)=2.21]$.

For the human observers, coherence values at performance levels of $80 \%$ and $90 \%$ correct were, on average, four times lower with a dot lifetime of 200 than with a dot lifetime of 5 . For the pigeons, on the other hand, coherence thresholds decreased, on average, by a factor of two.

The fact that the pigeons' coherence thresholds were lower with a dot lifetime of 200 than with a dot lifetime of 5 suggests that the pigeons integrated motion signals, albeit with an efficiency level less than that of humans. This evidence for motion integration is important, because it argues against the possibility that the pigeons solved the task with a mechanism that did not use any temporal integration of motion signals. For example, it is theoretically possible to solve the motion task by responding solely on the basis of the proportion of dots that move in a consistent direction across a single pair of frames. If the pigeons solved the task in this fashion, they should have shown no benefit from increasing the dot lifetime. Although the decreased thresholds in Experiment 2A, as compared with Experiment 1, are inconsistent with this possibility, the performance gain for the pigeons is small enough to warrant further investigation. A more direct assessment of the effect of dot lifetime is also justified because Experiments 1 and $2 \mathrm{~A}$ were separated by several months.

\section{EXPERIMENT 2B}

This experiment was designed to provide a more direct assessment of the effect of dot lifetime on pigeons' accuracy in detecting coherent motion. Sessions in which the dot lifetime of the dynamic random dot displays was set to 200 frames and 5 frames were alternated at three coherence levels.

\section{Method}

Subjects. The same pigeons as those used in Experiments 1 and $2 \mathrm{~A}$ were used in Experiment 2B. Pigeons 6 and C229 participated immediately after completing Experiment 1, whereas pigeon C201 participated immediately after completing Experiment $2 \mathrm{~A}$.

Procedure. For each bird, pairs of sessions were presented at three coherence levels. For one session of each pair, dot lifetime was set to 5 , and for the other session, dot lifetime was set to 200 , with order varying across pairs. For birds 6 and C229, coherence levels of 15, 20, and 25 were tested. For bird C201, coherence levels of 20,25 , and 30 were tested. In each case, coherence levels started at the higher value and decreased to the lower value. 


\section{Humans}
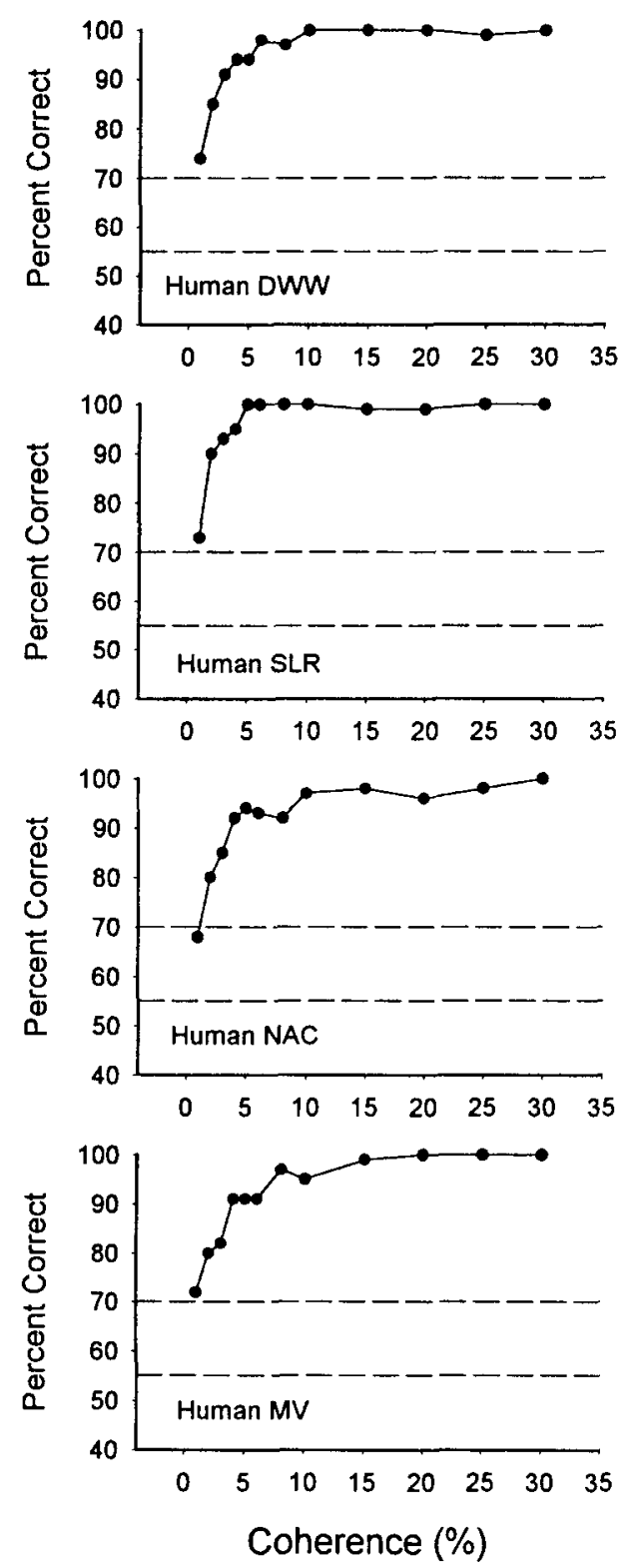

\section{Pigeons}
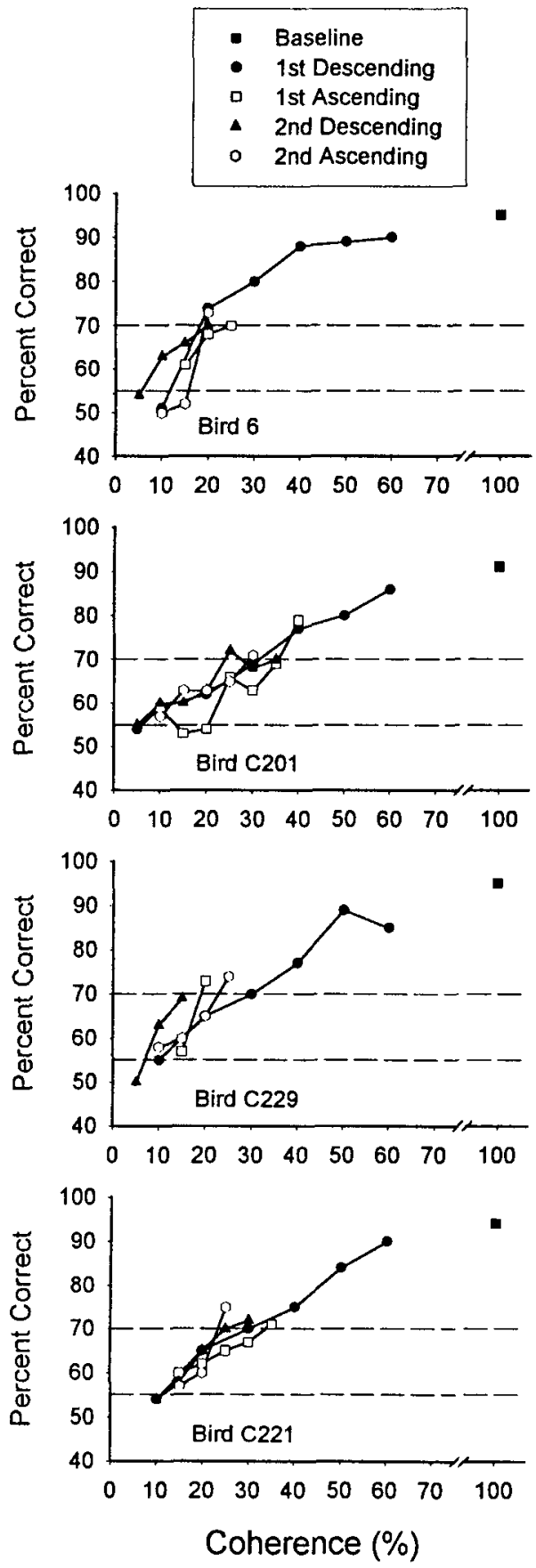

Figure 2. Coherence thresholds (see definition in text) for random dot patterns with a dot lifetime of 200 , for 4 human observers and 4 pigeon observers in Experiment $2 \mathrm{~A}$.

\section{Results and Discussion}

For all the statistical tests, the alpha level was set to .05 . Figure 3 shows accuracy as a function of coherence level for the 3 birds. On average, accuracy was $12 \%$ higher with a dot lifetime of 200 than with a dot lifetime of 5 . However, in order to interpret this performance change, an additional factor has to be taken into account- namely, the dependence of dot coherence on dot lifetime. With a dot lifetime of 5 frames, $20 \%$ of the coherently moving dots are moved to a new random position in every frame, and the effective dot coherence is, therefore, $20 \%$ lower than the notional dot coherence. With a dot lifetime of 200 , 

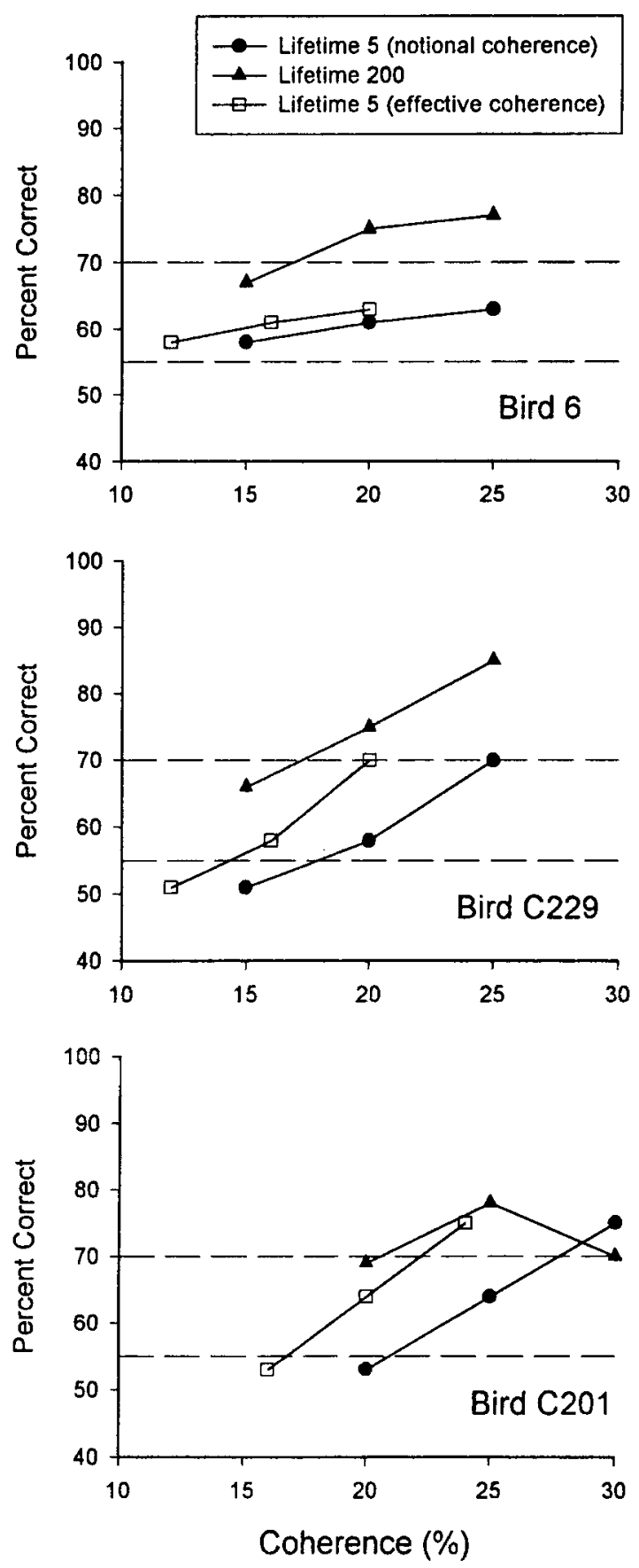

Figure 3. Accuracy scores at three levels of motion coherence for random dot patterns with a dot lifetime of 5 and a dot lifetime of $\mathbf{2 0 0}$ for 3 pigeons in Experiment 2B. Performance for a dot lifetime of 5 is shown as a function of notional and effective dot coherence (see definition in text).

only $0.5 \%$ of the coherently moving dots are moved to a new random position in every frame. With a notional dot coherence of $25 \%$, the effective dot coherence is, therefore, $20 \%$ for a dot lifetime of five and $24.875 \%$ for a dot lifetime of 200 .
Figure 3 also shows accuracy for a dot lifetime of 5, replotted in terms of effective dot coherence. In these terms, accuracy was, on average, about $6 \%$ higher with a dot lifetime of 200 than with a dot lifetime of 5. Similarly, with a dot lifetime of 200 , coherence could be reduced, on average, by about $9 \%$, to obtain the same performance level as obtained with a dot lifetime of 5 .

In order to provide statistical confirmation that accuracy was affected by lifetime, we compared performance at two effective coherence levels: $20 \%$ and $16 \%$. For $20 \%$ effective coherence, we used the accuracy scores obtained from the programmed coherence levels of $25 \%$ and $20 \%$ for the sessions with lifetimes of 5 and 200 , respectively. For $16 \%$ effective coherence, we used the accuracy scores obtained from the programmed coherence level of $20 \%$ for the sessions with lifetimes of 5 , and we conducted a linear interpolation of the accuracy scores obtained from $15 \%$ and $20 \%$ coherence levels for the sessions with a lifetime of 200. A two-way repeated measures ANOVA, with effective coherence (16 vs. 20 ) and lifetime (5 vs. 200 ), revealed significant main effects of both coherence $[F(1,2)=19.63]$ and lifetime $[F(1,2)=27.12]$ and no significant interaction $[F(1,2)=0.23]$.

The results of Experiments $2 \mathrm{~A}$ and $2 \mathrm{~B}$ indicate that increasing dot lifetime does decrease pigeons' coherence thresholds, albeit to a lesser extent than it does for human observers. The fact that the pigeons' motion sensitivity was significantly affected by lifetime indicates that their lower motion sensitivity, relative to that of humans, cannot be attributed solely to a lack of motion integration. If the pigeons had solved the task without using any motion integration, they should not have shown an effect of dot lifetime.

Although our results indicate that the pigeons did perform motion integration, this motion integration is clearly less efficient than that performed by humans. It should be noted that the correction for effective coherence level does not alter the conclusion that the humans benefitted more from an increase in lifetime than did the pigeons: Even with the correction applied, the coherence thresholds for the humans were four times lower, on average, with a dot lifetime of 200 than with a dot lifetime of 5 , whereas the pigeons' thresholds were only two times lower, on average.

The lower efficiency in motion integration by pigeons appears to occur at both the global and the local level. Evidence for less efficient global motion integration comes from the poorer motion sensitivity of pigeons than of humans when dot lifetime is 5 and little local integration is possible. Evidence for less efficient local motion integration comes from Experiments $2 \mathrm{~A}$ and $2 \mathrm{~B}$, which showed less benefit from increasing lifetime for pigeons than for humans. Psychophysical and neurophysiological evidence indicates that, for the primate visual system, local motion integration occurs at the level of V1 and MT, and global motion integration occurs at the level of MT and beyond (see, e.g., Simoncelli \& Heeger, 1998). This, in turn, suggests that the superiority of human motion per- 
ception should be attributed, at least in part, to integration mechanisms operating at the level of MT and beyond. This is further discussed below.

\section{EXPERIMENT 3}

For all the statistical tests, the alpha level was set to .05 . The results of Experiments 1 and $2 \mathrm{~A}$ indicated that pigeons' sensitivity in detecting coherent motion is substantially lower than that of humans. Specifically, the pigeons' coherence thresholds were at least eight times higher than those of the human observers. In those experiments, the motion signal was manipulated by altering the proportion of coherently moving dots. Another way to manipulate the motion signal, which should affect motion sensitivity, is to vary the velocity of motion. On a raster scan monitor, velocity cannot be manipulated directly, because motion is created by displacement of the dots rather than by direct movement of continuously illuminated dots. Consequently, there are two ways in which speed of movement can be manipulated. One is to hold frame update rate constant while varying spatial displacement of each dot. The second is to hold the spatial displacement constant while varying the frame update rate. Both means of manipulating velocity of motion also alter other aspects of the motion signal, but for the present purpose, this was not a concern. We varied velocity by altering screen update rate, while recognizing that this manipulation also would be expected to alter the strength of the motion signal. Our interest was in determining how detection of coherent motion by pigeons and humans would be affected by decreases in the velocity of motion via changes in frame update rate.

\section{Method}

Subjects. Two pigeons served as subjects, 1 with no motion stimuli experience (bird 809) and 1 (C229) that had previously served in motion coherence tests in Experiment $2 \mathrm{~A}$. Two of the authors and 1 naive subject served as human observers.

Stimuli. Dot velocity was manipulated by changing the rate at which the displays were updated. In Experiments 1,2A, and 2B, the screen update rate was $60 \mathrm{~Hz}$, corresponding to a velocity of $47.9 \mathrm{deg} / \mathrm{sec}$ for coherently moving dots. In this experiment, screen update rates were varied in the range of $5-60 \mathrm{~Hz}$, corresponding to velocities in the range of $4.2-47.9 \mathrm{deg} / \mathrm{sec}$ for coherently moving dots. It should be pointed out that the video refresh rate remained constant throughout all the conditions, and hence, there was no confounding of dot velocity and video flicker rate.

Procedure. For the pigeons, the coherence level of the positive stimulus was set to $100 \%$ for all the trials, and dot lifetime was set to 200 frames. The screen update rate used during training and baseline sessions was $60 \mathrm{~Hz}$-that is, dot velocity was $47.9 \mathrm{deg} / \mathrm{sec}$.

During the first series (first descending), the screen update rate of both the positive and the negative stimuli decreased across test sessions until accuracy in detecting the stimulus with coherent motion fell below $55 \%$ correct. These decreases were in steps of $15 \mathrm{~Hz}$ until the screen update rate reached $15 \mathrm{~Hz}$ (velocity, $12.7 \mathrm{deg} / \mathrm{sec}$ ) and then were in steps of $5 \mathrm{~Hz}$. During the second series (first ascending), the screen update rate was increased in steps of $5 \mathrm{~Hz}$ until accuracy rose above $70 \%$ correct. During the third series (second descending), the screen update rate was decreased in steps of $5 \mathrm{~Hz}$ until accuracy fell below 55\% correct. During the last series (sec- ond ascending), the screen update rate was increased in 5\% steps until accuracy rose above $70 \%$ correct.

For the human observers, performance in identifying coherent motion was measured with a dot lifetime of five at two coherence levels, $10 \%$ and $100 \%$, and for screen update rates of $5,10,15,30$, 45 , and $60 \mathrm{~Hz}$, corresponding to dot velocities of $4.2,8.5,12.7$, $25.1,36.9$, and $47.9 \mathrm{deg} / \mathrm{sec}$.

\section{Results and Discussion}

For all the statistical tests, the alpha level was set to .05 . Human performance in identifying coherent motion as a function of dot velocity for two levels of coherence, $10 \%$ and $100 \%$, is shown in the left panel of Figure 4 . As was expected from the results of the coherence experiments, performance for $10 \%$ coherence was clearly lower than performance for $100 \%$ coherence. For a coherence level of $10 \%$, the $70 \%$ velocity thresholds were $10.9,14.9$, and $14.8 \mathrm{deg} / \mathrm{sec}$, and 1 subject remained above the $55 \%$ threshold at all the velocities tested. For a coherence level of $100 \%$, accuracy was virtually perfect over the whole range of dot velocities tested.

The pigeons' performance in identifying coherent motion as a function of dot velocity at a coherence level of $100 \%$ is shown in the right panel of Figure 4 . The $70 \%$ velocity thresholds were 29.5 and $27.1 \mathrm{deg} / \mathrm{sec}$, and the $55 \%$ velocity thresholds were 8.5 and $10.6 \mathrm{deg} / \mathrm{sec}$, for the 2 pigeons, respectively.

The third panel of Table 1 shows mean latencies and accuracy scores, averaged across the two sessions that completed the ascending thresholds and the two sessions that completed the descending thresholds. As in the previous experiments, latencies were similar in the two types of sessions, despite the large differences in accuracy.

Once again, the pigeons were much poorer than the humans at discriminating coherent motion from dynamic noise. For the pigeons, $70 \%$ thresholds for velocity obtained with $100 \%$ coherent motion stimuli and a dot lifetime of 200 were in the range of $27-29 \mathrm{deg} / \mathrm{sec}$. For the humans, on the other hand, $70 \%$ thresholds for velocity with $10 \%$ coherent motion stimuli and a dot lifetime of 5 were in the range of $11-15 \mathrm{deg} / \mathrm{sec}$. When coherence was increased to $100 \%$ for the human observers, accuracy was virtually perfect at all the velocities tested. Clearly, the human motion system seems to saturate much more quickly than that of pigeons.

The velocity thresholds obtained with our random dot stimuli are substantially higher than those obtained by Hodos et al. (1975) with moving bars: The average $70 \%$ threshold for our pigeons $(28.3 \mathrm{deg} / \mathrm{sec})$ was more than five times higher than the average $75 \%$ threshold (4$6 \mathrm{deg} / \mathrm{sec}$ ) reported by Hodos et al. (1975). This difference may reflect the fact that, in Hodos et al.'s experiment, pigeons discriminated moving from static stimuli, whereas in our experiments, pigeons discriminated coherently moving stimuli from randomly moving stimuli. Furthermore, in Hodos et al.'s experiment, positional cues (i.e., detection of a change in spatial position of the moving stimulus) could be used for discrimination, but in our experiments, this was not possible, because changes in po- 


\section{Humans}

- Coherence 100

- Coherence 10
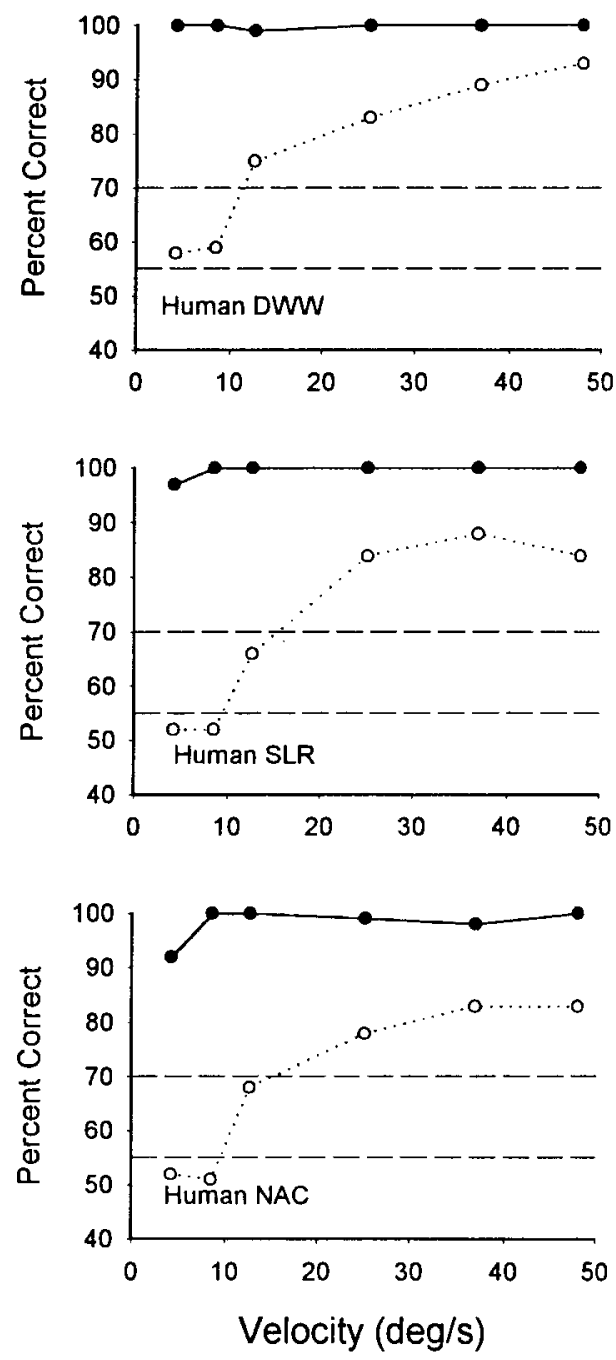

\section{Pigeons}
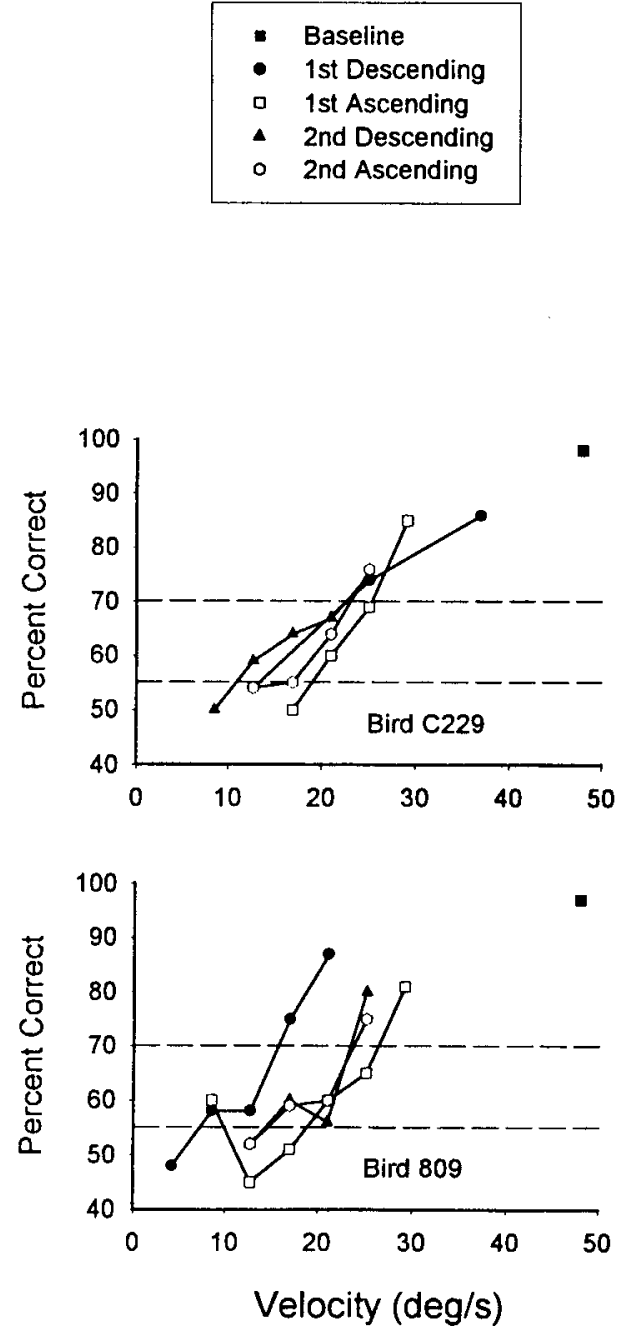

Figure 4. Velocity thresholds (see definition in text) for 3 human observers and 2 pigeons in Experiment 3, with coherence levels of $10 \%$ and $100 \%$ for humans and $100 \%$ for pigeons.

sition of the dots occurred within both the random and the coherent movement. Alternatively, our higher thresholds may reflect the fact that frame update rate not only changed the velocity of motion, but also weakened the motion signal.

\section{EXPERIMENT 4}

In each experiment that we have reported thus far, the performance of pigeons was found to be much poorer than that of humans. These differences in performance may be attributed to differences in the motion-processing systems of the two species, to some general performance inferiority of pigeons, relative to humans, on all visual discrimination tasks, or to the stimuli and methods that we used to investigate motion processing in pigeons in the three previous experiments. It seems unlikely that general performance deficits or problems with the stimuli or methods account for the high motion sensitivity thresholds of the pigeons, because their performance was very accurate on baseline conditions in which the motion discrimination was relatively easy. Nevertheless, it is impor- 
tant to demonstrate that pigeons can display thresholds that are more similar to those of humans on a visual discrimination not based on motion detection. Accordingly, we tested both pigeons and humans on another visual discrimination task involving the detection of (static) gratings in noise, using methods and stimuli that were highly similar to those used to study motion processing.

\section{Method}

Subjects. Three pigeons (6, C229, and 63) served as subjects. Bird 6 had previously participated in Experiments 1 and 2, bird C229 had previously participated in all three experiments, and bird 63 had not previously participated in any of the motion experiments. Two of the authors and two naive observers served as human subjects.

Apparatus and Stimuli. The apparatus was the same as that used in Experiments 1-3, and the grating stimuli were as described in the General Method section.

Procedure. Noise thresholds were determined for the pigeons by systematically decreasing and increasing noise levels in the gratings until accuracy either fell below $55 \%$ (descending series) or rose above $70 \%$ (ascending series), respectively. Each test session began with 10 warm-up trials in which the positive stimulus was a noisefree grating. During the first series (first descending), the noise level of the positive stimulus was increased in 5\% steps (starting from $0 \%$ noise) until the noise level reached $35 \%$, and then the noise level of the positive stimulus was increased in $2 \%$ steps (starting from $36 \%$ noise) across each test session until accuracy fell below $55 \%$ correct. During the second series (first ascending), the noise level was decreased in $2 \%$ steps until accuracy rose above $70 \%$ correct. During the third series (second descending), the noise level was increased in $2 \%$ steps until accuracy fell below $55 \%$ correct. During the last series (second ascending), the noise level was decreased in $2 \%$ steps until accuracy rose above $70 \%$ correct.

For the human subjects, detection accuracies were measured for noise levels in the range of $40 \%-49 \%$ in steps of $1 \%$.

For both the pigeons and the humans, two thresholds $(70 \%$ and $55 \%$ ) were estimated as was described in Experiment 1.

\section{Results and Discussion}

For all the statistical tests, the alpha level was set to .05 . The left panel of Figure 5 shows accuracy in detecting the gratings as a function of noise level for each of the 4 human observers. The $70 \%$ thresholds were $45.2 \%$, $44.8 \%, 44.6 \%$, and $44.9 \%$ noise, and the $55 \%$ thresholds were $47.1 \%, 47.1 \%, 45.9 \%$, and $45.9 \%$ noise, for the 4 observers, respectively.

The right panel of Figure 5 shows accuracy in detecting the gratings as a function of noise level for each of the 3 pigeons. The $70 \%$ thresholds were $37 \%, 39.9 \%$, and $41 \%$ noise, and the $55 \%$ thresholds were $43 \%, 45.8 \%$, and $45.9 \%$ noise, for the 3 pigeons, respectively.

The pigeons' performance did not vary systematically with orientation of the gratings (horizontal and vertical). Accuracy for each orientation was compared, using the two sessions that completed the ascending thresholds. No significant difference in accuracy across the two grating directions was found $[t(2)=0.10]$.

As in the motion detection experiments, the pigeons' latencies (time from onset of the grating display until the bird completed the two-peck response requirement) did not vary systematically with accuracy. The bottom panel of Table 1 shows the mean latencies and accuracy scores, averaged across the two sessions that completed the ascending thresholds and the two sessions that completed the descending thresholds. Despite differences in accuracy, latencies were not significantly different for the two types of sessions $[t(2)=2.70]$.

The pigeons' performance in discriminating gratings with varying noise levels was only marginally poorer than that of the humans. On average, the $70 \%$ thresholds were only approximately $6 \%$ higher for the pigeons than for the humans, and the $55 \%$ thresholds were less than $2 \%$ higher for the pigeons than for the humans. These results suggest that pigeons are not substantially inferior to humans in performance on all visual discrimination tasks, and, more important, they suggest that the methods and stimuli used in Experiments 1-4 are appropriate for investigating visual discrimination processes in pigeons. Thus, the dramatically inferior performance of the pigeons, relative to the humans, in the motion-processing experiments (Experiments 1-3) is most likely due to differences in motion-processing mechanisms, rather than to methodological inadequacies, since the same general methods used to study motion processing were used to study noise thresholds.

\section{GENERAL DISCUSSION}

Motion detection thresholds were determined for pigeons and humans, using random dot displays. It was found that pigeons have a much lower motion sensitivity and a much lower resilience to dynamic noise than do humans. Earlier studies have suggested the same, but we can provide much stronger support for this conclusion, given that our comparison of motion sensitivity in pigeons and humans used stimuli that were matched as closely as possible.

In Experiment 1, we found that coherence thresholds were about eight times lower for human than for pigeons. Thus, the pigeons in our study were comparatively poor at detecting coherent motion in the random dot displays, even though acquisition of the discrimination was rapid (all the birds showed accuracy levels of $80 \%$ or higher by their third training session). We suggest this difference is due to a weaker spatiotemporal integration mechanism in the pigeon visual system.

In Experiment 2, we investigated the effect of long dot lifetimes on motion detection. The pigeons showed a small but significant improvement in performance. This was confirmed in a subsidiary study in Experiment 2B. The finding that performance was affected by lifetime indicates that the pigeons did not solve the task without performing any motion integration. However, the finding that the benefit of increased lifetime was considerably smaller for pigeons than for humans indicates that pigeons' local motion integration is less efficient than that of humans. Thus, taken together, our results suggest that 
Humans
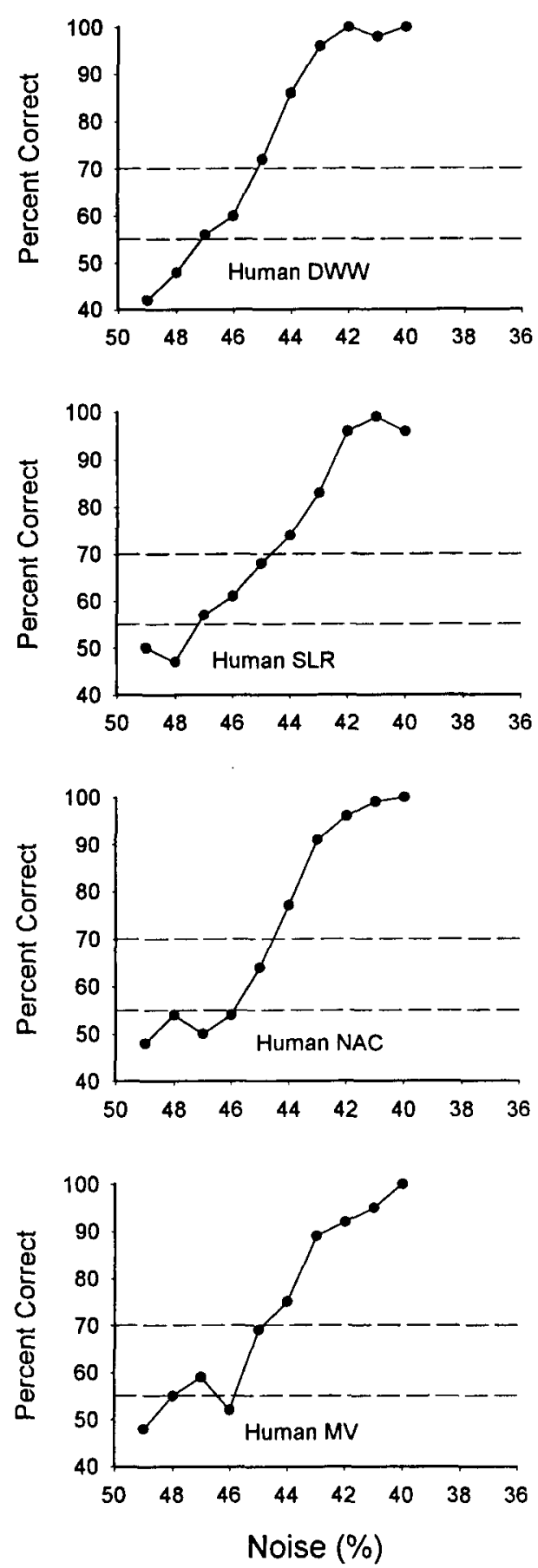

Pigeons
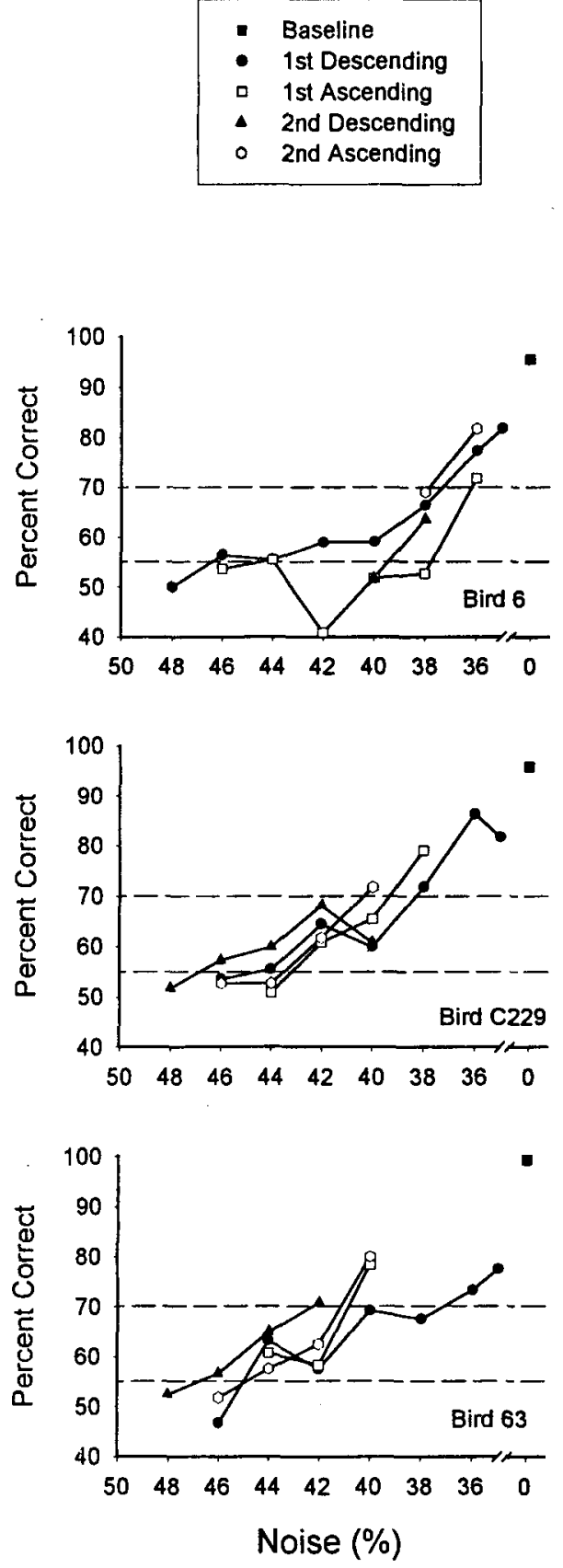

Figure 5. Noise thresholds for gratings (see definition in text), for 4 human observers and 3 pigeon observers in Experiment 4.

the inferior motion sensitivity of pigeons may be attributed to poorer motion integration at both the global and the local levels.

In Experiment 3, we investigated the effect of dot velocity on motion detection. It was found that velocity had a large effect on pigeons' ability to detect motion, but much less effect on humans. Even when humans were tested with lower levels of coherence ( $10 \%$ vs. $100 \%$ for pigeons) and with a dot lifetime of 5 instead of 200, motion detection thresholds were still approximately two times higher for pigeons than for humans. These results suggest that the human motion system saturates much 
more quickly above threshold, a conclusion that is compatible with the view that the human motion system has a much more efficient motion integration component.

Finally, in Experiment 4, we found that pigeons perform almost on par with humans on a static spatial discrimination task with stimuli and procedures that were highly similar to those used in the motion experiments. The considerably lower performance of pigeons in motion experiments can, therefore, not be simply attributed to methodological inadequacies but appears to be symptomatic of differences between the motion systems of humans and pigeons.

Because we could not control viewing distance in pigeons, owing to the nature of the response (i.e., pecking requires head movement), one might argue that the difference in motion detection thresholds for pigeons and humans could simply be due to differences in viewing distance. This seems highly unlikely for two reasons. First, our observations of the pigeons as they pecked the motion stimuli indicated low variability in the viewing distances. Almost invariably, the pigeon pecked the start stimulus and then stayed close to the screen and shifted left or right to peck the motion stimulus. Second, we conducted another series of tests with human subjects, under procedures that were identical to those of Experiment 1, but with stimuli that were $39 \%$ smaller than those used in the present experiments. This reduction in size is comparable with an increase in viewing distance of 14 to $24 \mathrm{~cm}$. The $70 \%$ coherence thresholds were $4.2 \%, 4.3 \%, 5.3 \%$, and $8 \%$ for 4 observers, almost identical to those reported in Experiment 1 . Thus, even if our matching of viewing distance was not exact, differences in viewing distance are unlikely to underlie the substantial differences in motion detection performance.

\section{Coherence in Random Dot Displays: \\ A Tool for Investigating Motion Pathways}

Efficient detection of motion direction in noisy random dot displays requires the spatial and temporal integration of local motion signals into a global motion percept. Using the same type of stimuli as those used in the present study, neurophysiological studies of the primate visual system have concluded that local motion detection is mediated by direction-selective cells in area $\mathrm{V} 1$ and that the spatial, temporal, and directional integration is achieved at the level of pattern cells in area MT (for a review, see Maunsell \& Newsome, 1987) and higher brain areas. Newsome and Paré (1988) found that lesions of area MT led to a strong elevation of coherence thresholds, from about $1 \%-7 \%$ (dependent on spatial displacement) to about $10 \%-50 \%$. Saito (1993) has shown that human performance in direction discrimination as a function of coherence is closely matched by directional response functions of type D cells in the dorsal part of area MST. Finally, some recent studies with Alzheimer patients (e.g., Hof, Bouras, Constantinidis, \& Morrison, 1989; Trick \& Silverman, 1991) suggest that the decreased motion sensitivity (i.e., the strongly elevated coherence thresholds) of these patients is due to disruption of the occipitoparietal pathway. Taken together, these results indicate that areas MT and MST play a crucial role in the spatiotemporal integration of motion signals. Although perception of coherent motion is possible without the functioning of area MT, maximal performance in primates, which is far superior to that of pigeons, may be due, at least in part, to the integration mechanisms operating at the level of area MT and beyond.

We believe similar studies could be performed in pigeons, to determine what structures might be important for spatiotemporal integration of motion signals. The avian visual system can be divided into three major afferent pathways: the thalamofugal system, the tectofugal system, and the accessory optic system (AOS; for a review, see Emmerton, 1983a; Frost, 1985; Frost et al., 1994). The thalamofugal and tectofugal systems are thought to correspond to the geniculostriate and tectopulvinarextrastriate pathways in mammals (Karten \& Shimizu, 1989). Given this, one would think that structures in the tectofugal system are likely candidates for spatiotemporal integration of local motion signals, but in all three avian visual pathways, the majority of neurons exhibit motion sensitivity. However, on the basis of the results of Experiment 3, we believe that the AOS was not involved in the processing of the stimuli used in the present experiment. In Experiment 3, we noted that the pigeons performed poorly on the task at velocities below about $10 \mathrm{deg} / \mathrm{sec}$. This finding is in agreement with the reported velocity sensitivities in the thalamofugal systems. For the thalamofugal system, Miceli et al. (1979) have reported that neurons in the visual wulst (the putative equivalent of the primary visual cortex) respond to small stimuli moving between 1 and $50 \mathrm{deg} / \mathrm{sec}$, although the average optimal velocity was not stated. In the tectofugal system, neurons are sensitive to small stimuli moving at velocities of between 2 and $300 \mathrm{deg} / \mathrm{sec}$ (Frost \& DiFranco, 1976; Hughes \& Pearlman, 1974; Wang \& Frost, 1991). However, Frost and DiFranco noted that few tectal cells had velocity preferences below $20 \mathrm{deg} / \mathrm{sec}$, and thus it is not surprising that the pigeons' performance was superior with the higher velocity stimuli. In the $A O S$, most neurons respond to moving large-field visual stimuli and prefer velocities in the range of $0.5-5 \mathrm{deg} / \mathrm{sec}$ (Morgan \& Frost, 1981). Thus, one might be surprised that the pigeons failed to distinguish motion from dynamic noise at slower velocities. However, Frost (1985) has emphasized that the AOS is specialized for visual motion resulting from selfmotion and not for the detection of object motion - that is, it responds to global optic flow patterns covering a large part of the visual field, rather than to spatially limited motion patterns arising from moving objects. Analysis of global motion within optic flow patterns also requires spatiotemporal integration of local motion signals, although over a much larger area than the stimuli used in the present study. It would be interesting to use the procedures of the present study to compare primates' and pigeons' responses to motion integration in simulations of 
optic flow patterns resulting from self-motion. The AOS is highly developed in both pigeons and primates, although, in primates, there is a massive input to the AOS area MST that seems related to the frontal position of the eyes (see, e.g., Grasse \& Cynader, 1990).

\section{Summary}

The present study improves on previous ones in two major respects. First, motion detection thresholds were determined for pigeons and humans, using stimuli that were matched as closely as possible for the two species. The present study, therefore, provides the most direct comparison of motion processing in pigeons and humans conducted to date. Moreover, our control experiment, which compared the performances of pigeons and humans on a static visual discrimination, allowed us to rule out the possibility that the differences obtained simply reflected general performance factors or methodological inadequacies. Second, the present study is the first to determine motion detection thresholds in pigeons, using dynamic random dot stimuli. We were thus able to study motion sensitivity in the absence of positional and/or form cues. This may also be the reason that we obtained higher velocity thresholds than did previous studies. Finally, our experiments suggest that it is likely that the inferior motion sensitivity of pigeons is due to poorer spatiotemporal motion integration.

\section{REFERENCES}

BEDELL, H. E., \& Johnson, C. A. (1995). The effect of flicker on foveal and peripheral thresholds for oscillatory motion. Vision Research, 35, 2179-2189.

Britto, L. R. G. D., Brunelli, M., Francesconi, W., \& Magni, F. (1975). Visual response pattern of thalamic neurons in the pigeon. Brain Research, 97, 337-343.

EMmerton, J. (1983a). Functional morphology of the visual system. In M. Abs (Ed.), Physiology and behavior of the pigeon (pp. 22 1-244). London: Academic Press.

Emmerton, J. (1983b). Vision. In M. Abs (Ed.), Physiology and behavior of the pigeon (pp. 245-266). London: Academic Press.

EMmERTON, J. (1986). The pigeon's discrimination of movement patterns (Lissajous figures) and contour-dependent rotational invariance. Perception, 15, 573-588.

Frost, B. J. (1985). Neural mechanisms for detecting object motion and figure-ground boundaries contrasted with self-motion detecting systems. In D. Ingle, M. Jeannerod, \& D. Lee (Eds.), Brain mechanisms and spatial vision (pp. 415-419). Dordrecht: Nijhoff.

Frost, B. J., \& DiFranCo, D. E. (1976). Motion characteristics of single units in the pigeon optic tectum. Vision Research, 16, 1229-1234.

Frost, B. J., Wylie, D. R., \& WANG, Y.-C. (1994). The analysis of motion in the visual systems of birds. In P. Green \& M. Davies (Eds.), Perception and motor control in birds (pp. 248-269). Berlin: SpringerVerlag.

Grasse, K. L., \& Cynader, M. S. (1990). The accessory optic system in frontal-eyed animals. In A. Leventhal (Ed.), Vision and visual dysfunction: Vol. IV. The neuronal basis of visual function (pp. 111-139). New York: MacMillan.

Hodos, W., Leibowitz, R. W., \& Bonbright, J. C., JR. (1976). Nearfield visual acuity of pigeons. Effects of head location and stimulus luminance. Journal of the Experimental Analysis of Behavior, 25, 129-141.

Hodos, W., Smith, L., \& Bonbright, J. C., JR. (1975). Detection of the velocity of movement of visual stimuli by pigeons. Journal of the Experimental Analysis of Behavior, 25, 143-156.

Hof, P. R., Bouras, D., Constantinidis, J., \& Morrison, J. H. (1989).
Balint's syndrome in Alzheimer's disease: Specific disruption of the occipitoparietal pathway. Brain Research, 493, 368-375.

HuĞHes, C. P., \& Pearlman, A. L. (1974). Single unit receptive fields and the cellular layers of the pigeon optic tectum. Brain Research, 80, $365-377$.

JohanNSON, G. (1978). Visual event perception. In R. Held, H. W. Leibowitz, \& H.-L. Teuber (Eds.), Perception (pp. 675-711). Berlin: Springer-Verlag.

KARTEN, J. K., \& Shimizu, T. (1989). The origins of neocortex: Connections and lamination as distinct events in evolution. Journal of Cognitive Neuroscience, 1, 291-301.

Martinoya, C., \& Delius, J. D. (1990). Perception of rotating spiral patterns by pigeons. Biological Cybernetics, 63, 127-134.

Martinoya, C., Rivaud, S., \& Bloch, S. (1983). Comparing frontal and lateral viewing in the pigeon: II. Velocity thresholds for movement discrimination. Behavioural Brain Research, 8, 375-385.

MAUnSELL, J. H. R., \& Newsome, W. T. (1987). Visual processing in monkey extrastriate cortex. Annual Review of Neuroscience, 10, 363401.

Miceli, D., Gioanni, H., Reperant, J., \& Peyrichoux, J. (1979). The avian wulst: I. An anatomical study of afferent and efferent pathways. II. An electrophysiological study of the functional properties of single neurons. In A. M. Granda \& J. H. Maxwell (Eds.), Neural mechanisms of behavior in the pigeon (pp. 223-254). New York: Plenum.

Morgan, B., \& Frost, B. J. (1981). Visual response characteristics of neurons in nucleus of basal optic root of pigeons. Experimental Brain Research, 42, 181-188.

NAKAYAMA, K. (1985). Biological image motion processing: A Review. Vision Research, 25, 625-660.

Nakayama, K., \& Tyler, C. W. (1981). Psychophysical isolation of movement sensitivity by removal of familiar position cues. Vision Research, 21, 427-433.

NeWsome, W. T., \& PARÉ, E. B. (1988). A selective impairment of motion perception following lesions of the middle temporal visual area (MT). Journal of Neuroscience, 8, 2201-2211.

PELLI, D. G. (1990). The quantum efficiency of vision. In C. Blakemore (Ed.), Vision: Coding and efficiency (pp. 3-24). Cambridge: Cambridge University Press.

Pisacreta, R., \& WitT, K. (1985). Movement as the discriminative stimulus dimension in several conditional discriminations. Psychological Record, 35, 113-123.

PowELL, R. W. (1967). The pulse-to-cycle fraction as a determinant of critical flicker fusion in the pigeon. Psychological Review, 17, 151160.

SAITo, H.-A. (1993). Hierarchical neural analysis of optical flow in the macaque visual pathway. In T. Ono, L. R. Squire, M. E. Raichle, D. I. Perrett, \& M. Fukuda (Eds.), Brain mechanisms of perception and memory (pp. 121-140). Oxford: Oxford University Press.

Scase, M. O., Braddick, O. J., \& Raymond, J. E. (1996). What is noise for the motion system? Vision Research, 36, 2579-2586.

SIEGEL, R. K. (1970). Apparent movement detection in the pigeon. Journal of the Experimental Analysis of Behavior, 14, 93-97.

SiEgEL, R. K. (1971). Apparent movement and real movement detection in the pigeon: Stimulus generalization. Journal of the Experimental Analysis of Behavior, 16, 189-192.

SimonCElli, E. P., \& HeEger, D. J. (1998). A model of neuronal responses in visual area MT. Vision Research, 38, 743-761.

SNOWDEN, R. J., \& BRADDICK, O. J. (1989). Extension of displacement limits in multiple-exposure sequences of apparent motion. Vision Research, 29, 1777-1787.

Trick, G. L., \& Silverman, S. E. (1991). Visual sensitivity to motion: Age-related changes and deficits in senile dementia of the Alzheimer type. Neurology, 41, 1437-1440.

WANG, Y.-C., \& Frost, B. J. (1991). Visual response characteristics of neurons in the nucleus isthmi magnocellularis and nucleus isthmi parvocellularis of pigeons. Experimental Brain Research, 87, 624-633.

WATson, A. B. (1986). Temporal sensitivity. In K. R. Boff, L. Kaufman, \& J. P. Thomas (Eds.), Handbook of perception and performance (Vol. 1, pp. 6-1 to 6-43). New York: Wiley.

(Manuscript received March 31, 1998; revision accepted for publication August 31, 1998.) 